\title{
Disziplinäre Entdifferenzierung durch Leistungsbewertung? Der Fall Geschichtswissenschaft in Großbritannien
}

Julian Hamann, Humboldt University Berlin

\section{Zusammenfassung}

Der Beitrag fragt, ob systematische Forschungsassessments mit einer Entdifferenzierung disziplinärer Kulturen einhergehen. Der Anwendungsfall für diese Frage ist das britische Research Excellence Framework (REF). Es wird gezeigt, dass geschichtswissenschaftliche Departments in den vom Assessment prämierten oberen Ranggruppen vor allem Aufsätze in high impact-Fachzeitschriften publizieren, während die nicht vom Assessment ausgezeichneten Departments vor allem Sammelbandbeiträge publizieren. Der Beitrag kommt zu dem Schluss, dass eine generisch auf alle Disziplinen angewendete und symbolisch wie materiell wirkmächtige Leistungsbewertung zumindest hinsichtlich der Publikationsaktivitäten mit einer Entdifferenzierung disziplinärer Kulturen einhergeht.

\begin{abstract}
The contribution examines whether systematic research assessments go hand in hand with a dedifferentiation of disciplinary cultures. The case of application for this question is the British Research Excellence Framework (REF). The analysis reveals that history departments in the upper rank groups of the REF publish first and foremost articles in high impact journals, while those departments that are not rewarded by the assessment publish mainly contributions to edited volumes. The contribution concludes that research assessments that are generically applied across disciplines and that are both symbolically and materially efficacious go hand in hand with a dedifferentiation of disciplinary cultures in terms of publication activities.
\end{abstract}

\section{Einleitung}

Der vorliegende Beitrag widmet sich der Frage, ob es einen Zusammenhang gibt zwischen der mittlerweile fest etablierten Bewertung von Forschungsleistungen in systematisch angelegten Forschungsassessments und der - auch als Konvergenz beschreibbaren - Entdifferenzierung wissenschaftlicher Disziplinen. Motiviert ist diese Frage durch die in der Wissenschafts- und Hochschulforschung recht verbreitete und empirisch gut belegte Annahme der Reaktivität von Leistungsmessungen (Campbell 1979; Espeland \& Sauder 2007). Reaktivität meint hier, dass 
Universitäten wie auch einzelne Forscher*innen ihre Aktivitäten zumindest oberflächlich an eben jene Kriterien anpassen, die im Rahmen der Bewertung angelegt werden. Ziel einer solchen Anpassung ist die positive Beeinflussung der Bewertung und ein möglichst gutes Abschneiden im jeweiligen Wettbewerb (Krücken et al. 2021). Die strategische Antizipation der Bewertung kann von buchstäblich spielerischen Ansätzen des gaming bis hin zu wissenschaftlichem Fehlverhalten reichen (Biagioli et al. 2019). Dann wird Forschung rhetorisch gelabelt und verpackt, Ressourcen innerhalb der Universität werden gezielt umverteilt, Aufgaben werden strategisch umbenannt.

Vor dem Hintergrund dieser Annahme arbeitet mein Beitrag mit zwei Prämissen: Erstens wird davon ausgegangen, dass die strategische Antizipation von Bewertungskriterien umso wahrscheinlicher wird, je wirkmächtiger das Bewertungsverfahren ist. Mit anderen Worten: Nicht jedes Ranking hat einen gleichermaßen großen Einfluss auf die gerankten Objekte, nicht jedes Rating lädt gleichermaßen zu einem strategischen Umgang mit seinen Kriterien ein. Doch je mehr in einem Bewertungsverfahren symbolisch und materiell auf dem Spiel steht, je mehr Anerkennung oder Geld zur Disposition steht, desto höher ist der Anreiz, sich den zugrundeliegenden Kriterien strategisch anzupassen.

Die zweite Prämisse des Beitrags ist, dass Leistungsbewertungen umso eher zu einer Entdifferenzierung von Disziplinen führen, je generischer sie angewendet werden. Je systematischer und großflächiger also die Kriterien eines Forschungsassessments quer über alle Disziplinen angelegt werden, desto höher ist die Wahrscheinlichkeit, dass sich disziplinäre Kulturen unter dem Druck der Leistungsbewertung einander angleichen - und zwar in Richtung jener Kriterien, die bei der Bewertung in Anschlag gebracht werden.

Diesen beiden Prämissen geht der Beitrag im Folgenden empirisch nach. Dabei beziehe ich mich auf Datenmaterial, das ich in einer Studie zum britischen Forschungsassessment, dem Research Excellence Framework (REF), gesammelt habe. Der disziplinäre Anwendungsfall ist die Geschichtswissenschaft. Ziel des Beitrags ist es empirisch zu prüfen, ob es im Rahmen des REF zu einer Entdifferenzierung der Geschichtswissenschaft kommt, und zwar insbesondere zu einer Entdifferenzierung der geschichtswissenschaftlichen Publikationskultur.

Der Beitrag ist wie folgt aufgebaut: Auf eine Darstellung des REF und seiner grundlegenden Funktionsweise und Logik folgt ein Versuch, sich der Publikationskultur der Geschichtswissenschaft zu nähern. Der Hauptteil geht der Frage nach, inwiefern es im Rahmen des REF zu einer Entdifferenzierung dieser Publikationskultur kommt. Abschließend diskutiere ich die Frage, inwiefern generisch angewendete Verfahren der Leistungsbewertung als Motoren für disziplinäre Entdifferenzierung gelten können. 


\section{Funktionsweise und Logik des Research Excellence Framework}

Das bis 2008 Research Assessment Exercise (RAE) genannte REF ist eine groß angelegte Bewertung der Forschungsleistungen britischer Universitäten und ihrer Departments. Das RAE/REF wird seit 1986 regelmäßig etwa alle drei bis sieben Jahre durchgeführt. Damit ist es nicht nur eines der ältesten Forschungsassessments der Welt, sondern auch eines der am besten erforschten. Im Fokus der Forschung zum RAE/REF stehen etwa die durch das Verfahren veränderten Autoritätsstrukturen (Whitley et al. 2010) zwischen bewertenden und bewerteten Peers (Sharp \& Coleman 2005), zwischen Forschungs- und Lehrpersonal (Tapper \& Salter 2002) oder zwischen Universitätsmanagement und Departments (Yokoyama 2006). Untersucht wurden auch dysfunktionale Effekte des RAE/REF, wozu die inhaltliche Standardisierung etwa der Wirtschaftswissenschaften (Lee et al. 2013) oder der Rechtswissenschaft (Campbell et al. 1999) sowie die Stratifikation des akademischen Feldes in so genannte Elite- und Masseneinrichtungen gehören (Münch \& Schäfer 2014). Nicht zuletzt in Reaktion auf diese Kritik ist das RAE/REF über die Jahre hinweg immer weiter entwickelt worden (Stern 2016), so dass es mittlerweile wohl eines der weltweit avanciertesten und aufwändigsten Verfahren zur Bewertung von Forschungsleistung ist (Sivertsen 2017). Die folgende Darstellung des Verfahrens konzentriert sich schwerpunktmäßig auf das zuletzt durchgeführte REF 2014 - die nächste Runde des REF wird zum Zeitpunkt, zu dem dieser Beitrag verfasst wird, durchgeführt.

Organisiert wird das REF von den englischen, schottischen, walisischen und nordirischen Forschungsfördereinrichtungen, die zu Beginn jedes Assessments die disziplinären Einheiten der Bewertung festlegen. Diese units of assessment (UoA) genannten Einheiten sind gewissermaßen die disziplinären Schubladen, in die zu bewertende Forschung einsortiert werden muss. Das ist so lange unproblematisch, wie sich Forschung innerhalb der Grenzen größerer Disziplinen bewegt: „Soziologie“, "Geschichtswissenschaft“ oder „Physik“ sind im REF immer eigene UoAs gewidmet. Aber natürlich lässt sich Forschung nicht immer in dieses institutionelle Raster einsortieren. Kunsthistoriker*innen mussten sich im REF 2014 zum Beispiel entscheiden, ob sie in der UoA „Art and Design: History, Practice and Theory" oder in der UoA "History" bewertet werden.

Der Versuch, disziplinäre Strukturen in Form von UoAs institutionell zu spiegeln, ermöglicht bereits eine erste Einsicht zur Entdifferenzierung von Fächern durch Verfahren der Leistungsbewertung: Weder die genauen Umrisse der UoAs noch ihre Zahl bleibt über die verschiedenen Assessments hinweg stabil. Für die fünf seit 1992 durchgeführten Assessments lässt sich erkennen, dass die Zahl der UoAs deutlich gesunken ist (vgl. Abbildung 1). Die disziplinären Einheiten des RAE/REF sind offenbar immer größer und umfassender geworden. 
Abbildung 1: Zahl der UoAs im RAE/REF, 1992-2014

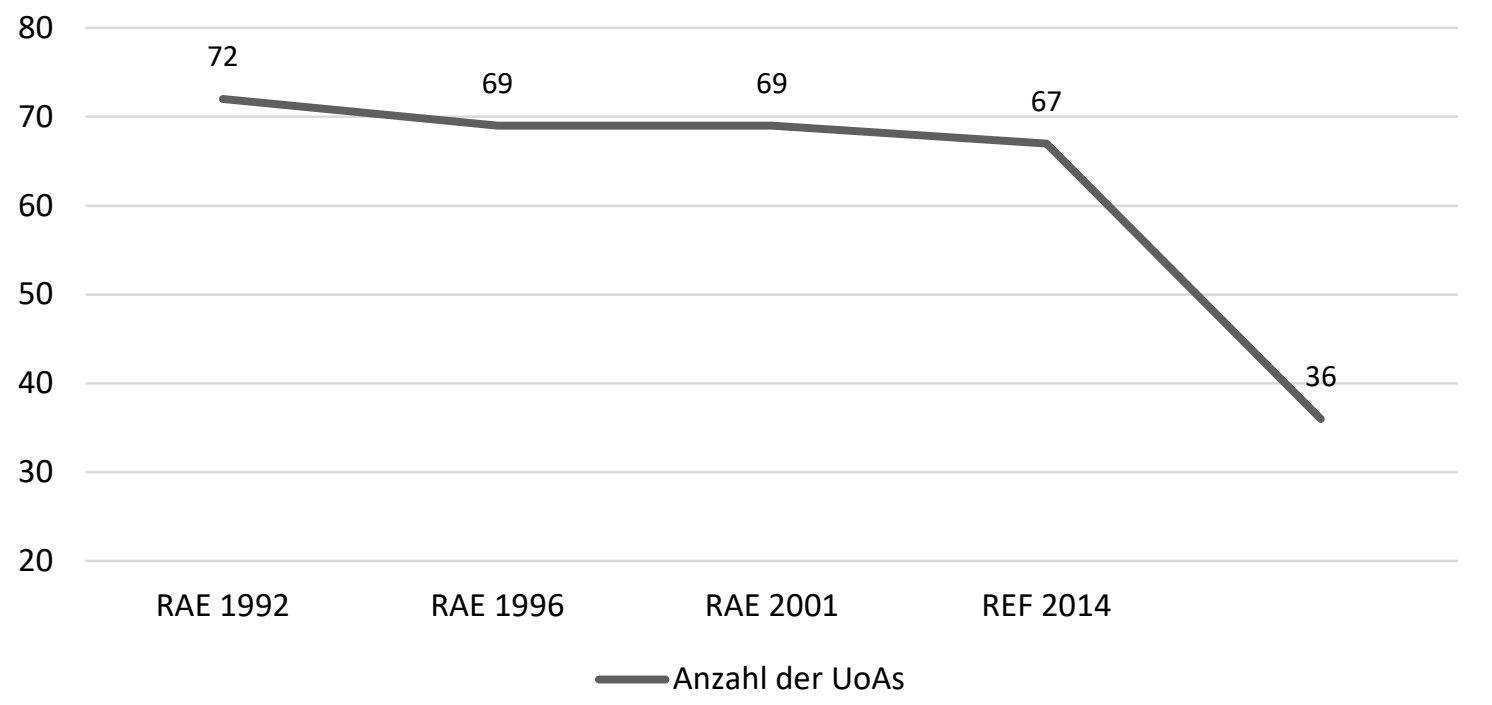

UoA = unit of assessment $\mathrm{RAE}=$ Research Assessment Exercise; REF $=$ Research Excellence Framework (eigene Berechnung nach RAE 1992; RAE 1996; RAE 2001b; REF 2014c)

Im weiteren Ablauf des REF müssen sich Departments entscheiden, in welcher UoA sie ihre Forschung einreichen wollen und auf die Arbeiten welcher Personen im Department dabei zurückgegriffen wird. Das im Zuge dieser Entscheidung von den Departments nominierte Forschungspersonal kann dann maximal vier Publikationen pro Person für einen Berichtszeitraum von sechs Jahren einreichen. ${ }^{1}$ Für jede UoA wird ein fachspezifisches Bewertungspanel eingerichtet, das aus peers im jeweiligen Fachgebiet besteht. Panels bewerten und benoten die eingereichten Publikationen, aggregieren die Noten auf Departmentebene und klassifizieren anhand dessen Departments in der jeweiligen UoA. ${ }^{2}$

Dass überhaupt fachspezifische Bewertungspanels eingerichtet werden, die eingereichte Publikationen qualitativ bewerten, zeigt bereits, dass das REF durchaus sensibel für fachkulturelle Differenzen ist. Dennoch folgt die Leistungsbewertung im REF einem standardisierten Format und seine Erhebungs- und Berichtszeiträume folgen einem einheitlichen Rhythmus - der Berichtszeitraum für einzureichende Publikationen beträgt für Physik, Geschichtswissenschaft und Philosophie

\footnotetext{
${ }^{1}$ Für die im REF 2021 durchgeführten Durchgänge soll die Differenzierung in Forschungs- und Lehrpersonal fallen gelassen werden, so dass Forschungsarbeiten von allen Mitgliedern eines Departments eingereicht werden können.

${ }^{2}$ Seit 2014 werden Departments nicht nur anhand ihrer research output genannten Publikationen, sondern auch anhand des societal impact ihrer Forschung sowie hinsichtlich ihrer institutionellen Forschungsumgebung bewertet. Der vorliegende Beitrag konzentriert sich auf die Bewertung von research output, die anteilsmäßig weiterhin den höchsten Stellenwert einnimmt. Dass es auch bei der Bewertung des societal impact von Forschung zu einer Entdifferenzierung disziplinärer Kulturen kommt, ist zumindest nicht unplausibel (vgl. Derrick \& Samuel 2016; Hamann et al. 2019).
} 
gleichermaßen sechs Jahre. Nicht zuletzt erfolgt die Bewertung von Publikationen zwar zunächst in einem offenen Peer Review-Verfahren, wird dann aber in allen UoAs in ein einheitliches Notensystem überführt. Dieses Notensystem zeigt, dass es letztlich für alle Disziplinen um originality, significance und rigour geht; die graduellen Noten unterscheiden dann Forschung nach "world-leading", "internationally excellent", "recognized internationally", "recognized nationally" und "unclassified" (REF 2012). So ausgeprägt die Sensibilität für fachkulturelle Differenzen auch sein mag, die Wirkmächtigkeit des REF besteht nicht zuletzt darin, dass das Verfahren diese Differenzen letzten Endes einebnet. Der forschungspolitische Reiz des Verfahrens liegt gerade darin, in so unterschiedlichen Disziplinen und Fachgebieten wie der Geschichtswissenschaft, der Physik und den Business and Management Studies „world leading research" zu identifizieren und prämieren.

Die Prämierung der bewerteten Departments und Universitäten ist der letzte Schritt des Verfahrens. Die Ergebnisse des REF werden medial sehr breit rezipiert und genießen eine hohe öffentliche Aufmerksamkeit. Für die besonders gut bewerteten Departments und Universitäten gehen damit erhebliche Prestigegewinne einher. Entscheidend für die Wirkmächtigkeit des REF ist weiterhin, dass das Bewertungsverfahren auch über die Verteilung materieller Ressourcen entscheidet. Auf Grundlage der Ergebnisse des REF 2014 wurden etwa zwei Milliarden Britische Pfund pro Jahr an die bewerteten Universitäten verteilt - in England beläuft sich die Summe auf fast 1,1 Milliarden Britische Pfund, was über 80\% der insgesamt kompetitiv vergebenen Fördergelder ausmacht (Technopolis Group 2018: 31). Es bleibt dann den Universitäten selbst überlassen, wie sie das Geld zwischen den Departments verteilen. Die genaue Allokation der Fördergelder ist weder für einzelne Universitäten noch für die jeweiligen Notenstufen bekannt. Vermutet wird, dass $75 \%$ der gesamten im REF zur Disposition stehenden Fördersumme auf die beste Notenstufe (,world leading research“) und 25\% auf die zweitbeste Notenstufe (,internationally excellent research“) verteilt wird (The Guardian 2014). Sicher ist, dass die Verteilung der Gelder ganz bewusst auf die Spitze „exzellenter" Forschung konzentriert ist:

"The UK is also one of the few countries where the assessment of the research quality aims at rewarding 'excellence' and the concentration of resources on the 'best' research wherever it is found. In the $\mathrm{RAE} / \mathrm{REF}$, a non-linear calculation of the institutional funding allocations is used, purposely skewing rewards towards those with the strongest performance." (Stern 2016: 49)

Diese hochselektive Allokation von Forschungsgeldern ist einerseits weniger dramatisch, als es auf den ersten Blick scheint: Im REF 2014 wurden immerhin drei Viertel der bewerteten Forschung für „world leading" oder "internationally leading" befunden und mit den beiden besten Noten ausgezeichnet (REF 2014b) - eine Inflation, die sich übrigens schlecht mit der nullsummenbasierten Exzellenzrhetorik vieler Forschungsassessments verträgt (Marginson 2015). Andererseits geht dennoch über die Hälfte der verteilten Forschungsgelder an die zehn drittmittelstärksten Universitäten (Technopolis Group 
2018: 36). Das macht die Schärfe des vom REF inszenierten Drittmittelwettbewerbs deutlich und entspricht der oben zitierten Verteilungslogik, die auf eine starke Konzentration der Ressourcen an der Spitze abzielt.

Die beiden Prämissen des Beitrags sind also gegeben: Erstens wird das REF generisch über alle Disziplinen hinweg angewendet, zweitens ist seine symbolische und materielle Wirkmächtigkeit enorm. Interessant für die Fragestellung des Beitrags ist nun das mission statement des REF. Hier wird betont, dass „identifying excellence in the rich diversity of research” das Ziel sei, und dass es darum gehe, „not to privilege any journal or conference rankings/lists, the perceived standing of the publisher or the medium of publication, or where the research output is published." (REF 2012) Mit anderen Worten: Es geht im REF darum, Exzellenz in der Vielfalt zu entdecken, weder der Ort noch die Art der Veröffentlichung sollen bei der Bewertung von Forschungsleistung eine Rolle spielen. Auf den ersten Blick verspricht das eine hohe Sensibilität für fachkulturelle Besonderheiten, wie sie sich etwa in Publikationskulturen ausdrücken.

\section{Die Publikationskultur der Geschichtswissenschaft - eine Annäherung}

Publikationskulturen sind ein guter Indikator für fachkulturelle Besonderheiten. Weil disziplinäre Eigenheiten, erst Recht solche in den Sozial- und Geisteswissenschaften, in der Wissenschafts- und Hochschulforschung nur unzureichend erforscht sind, muss sich eine Annäherung an die Publikationskultur der Geschichtswissenschaft auf vereinzelte Studien und anekdotische Evidenzen stützen. Dazu gehört, dass Unterschiede in den Publikationskulturen geschichtswissenschaftlicher Teilbereiche übergangen werden müssen - so kann ich nur vermuten, dass deutschsprachige Monografien in der Alten Geschichte wichtiger sind als in der Wissenschaftsgeschichte, und dass Letztere stärker auf englischsprachige Fachzeitschriftenartikel fokussiert ist. Auf solche innerdisziplinären Unterschiede kann im Folgenden nicht näher eingegangen werden.

Insgesamt und im Vergleich zu anderen Fächern kann einerseits festgestellt werden, dass die Monografie in der Geschichtswissenschaft einen hohen Stellenwert hat. Die Alexander von HumboldtStiftung hat 2009 ein Diskussionspapier zum Publikationsverhalten in unterschiedlichen Disziplinen herausgebracht, in dem Fachvertreter*innen über die Publikationskulturen in ihren Fächern berichten. Darin schreibt der Dresdner Althistoriker Martin Jehne (2009: 59): „Im Zentrum der Publikationsleistungen von Historikerinnen und Historikern steht nach wie vor das Buch. [...] Jede Stellenbesetzungs- oder Förderungskommission sieht sich bei Bewerbern zunächst einmal an, was an Büchern vorliegt.“ Zu Fachzeitschriften und Sammelbandbeiträgen heißt es weiter: 
„Aufsätze in Fachzeitschriften sind wichtig, doch hat sich das Publikationsverhalten in den letzten 20 Jahren erheblich zu Sammelbänden hin verschoben, und der Trend geht weiter in diese Richtung. [...] Ein historischer Beitrag zu einem Sammelband steht in der Wertigkeit zunächst einmal auf derselben Stufe wie ein Zeitschriftenaufsatz, es gibt keine konsequente Praxis, innovativere Beiträge für Zeitschriften zu reservieren und die Variation des schon Vertrauten in die Sammelbände zu verschieben." (Jehne 2009: 59)

Dieser augenscheinlich erfahrungsbasierten Perspektive aus der Alten Geschichte lässt sich eine bereits 1991 geäußerte Diagnose gegenüberstellen, die auf einer systematischen Erhebung von Publikationsmustern in der Germanistik, Anglistik und Geschichtswissenschaft beruht. Auf Grundlage dieser Erhebung wird ein "Siegeszug des wissenschaftlichen Artikels als vorherrschende Veröffentlichungsform“ in den genannten Disziplinen von den 1950ern bis zu den 1980ern konstatiert, auch erkennt man den „Aufstieg des Sammelbandes zu einer zahlenmäßig bedeutsamen Publikationsform." (Weingart et al. 1991: 283) Weiterhin ließe sich, gerade mit Blick auf die Geschichtswissenschaft, „das Argument nicht mehr aufrechterhalten, die Monographie sei die [...] typische Form der Publikation." (Weingart et al. 1991: 284-285) Tatsächlich zeigen die Autor*innen der Studie in einer Verlaufsperspektive von den 1950ern bis in die 1980er für die Geschichtswissenschaft einen starken Anstieg der pro-Kopf Publikation von Fachzeitschriftenaufsätzen, während sich die Monografie auf einem niedrigen Niveau einpendelt (Weingart et al. 1991: 289).

Die auf den ersten Blick unterschiedlichen Befunde lassen sich in zweierlei Weise vereinbaren: Zum einen ist denkbar, dass in der Geschichtswissenschaft eine doppelte Publikationskultur existiert, bei der Monografien wichtig für die breite, öffentliche Rezeption zentraler Themen sind, während die fachinterne Diskussion spezifischer Themen immer auch über Fachzeitschriftenaufsätze erfolgt. ${ }^{3}$ Zum anderen erklären sich die unterschiedlichen Befunde möglicherweise damit, dass Jehne vom Wert von Publikationsformen spricht, während Weingart et al. die Häufigkeit erhoben haben. Die Monografie kann also nach wie vor eine wichtige Publikationsform sein, auch wenn mittlerweile deutlich mehr Fachzeitschriftenaufsätze publiziert werden. In diese Richtung deutet auch die Studie von Björn Hammarfelt und Sarah de Rijcke, die auf Grundlage einer Umfrage unter Geisteswissenschaftler*innen in Schweden zu dem Schluss kommen, dass die Monografie weiterhin einen hohen Status habe, aber deswegen nicht die häufigste Publikationsform sei: „The status of the monograph is indisputable in many fields [...]. [A] distinctive feature of many research fields in the humanities is that the most 'important channel' is not by far the most common one." (Hammarfelt \& de Rijcke 2015: 7) In der Umfrage der Autor*innen zum Stellenwert unterschiedlicher Publikationsformen ist die Monografie dann auch dicht gefolgt vom peer review-Fachzeitschriftenaufsatz.

Zur spezifischen Publikationskultur der Geschichtswissenschaft lässt sich also vorsichtig resümieren, dass Monografien und auch Sammelbandbeiträge zumindest nicht weniger wichtig als

\footnotetext{
${ }^{3}$ Für diesen Hinweis danke ich einer*einem anonymen Gutachter*in.
} 
Fachzeitschriftenaufsätze zu sein scheinen. Was passiert mit einer solchen Publikationskultur, wenn sich Historiker*innen, so wie das in Großbritannien der Fall ist, seit Jahrzehnten in einer Prüfungs- und Bewertungssituation befinden, in der sie die „Exzellenz“ ihrer Forschung in einem einheitlichen Format nachweisen müssen?

\section{Entdifferenzierung der geschichtswissenschaftlichen Publikationskultur im Research Excellence}

\section{Framework?}

Wie oben argumentiert gelten für das REF die beiden Prämissen, nach denen eine Entdifferenzierung der geschichtswissenschaftlichen Publikationskultur wahrscheinlich ist: Das Forschungsassessment wird generisch über alle Disziplinen hinweg angewendet und es steht symbolisch wie materiell viel auf dem Spiel. Es liegt also nahe, dass für britische Historiker*innen hohe Anreize bestehen, das REF-Spiel mitzuspielen. Sofern das der Fall ist, müsste man eine Korrelation zwischen den von geschichtswissenschaftlichen Departments zur Bewertung eingereichten Publikationen und den im REF gebildeten Ranggruppen von Departments sehen. Die Departments, die ihr Publikationsverhalten an die generischen Bewertungskriterien anpassen, würden dann mit hohen Rangplätzen belohnt. Dieser Vermutung wird nun empirisch nachzugehen sein.

Werfen wir zunächst einen Blick auf die Ranggruppen in der Geschichtswissenschaft in den drei letzten Durchgängen des RAE/REF in den Jahren 2001, 2008 und 2014. Links sind die Top 6 und die Top 14 geschichtswissenschaftlicher Departments des jeweiligen Assessments abgebildet, rechts die Bottom 6 und Bottom 14 (Tabelle 1). ${ }^{4}$

\footnotetext{
${ }^{4}$ Die Ranggruppen Top 6, Top 14, Bottom 6 und Bottom 14 sind absichtlich beliebig gebildet - und nicht beliebiger als alternative Ranggruppen wie Top 5 oder Top 10.
} 
Tabelle 1: Top 6, Top 14 und Bottom 6, Bottom 14 geschichtswissenschaftlicher Departments im RAE/REF 2001, 2008, 2014

\begin{tabular}{|c|c|c|c|c|c|}
\hline \multicolumn{3}{|c|}{ Top 6/14 } & \multicolumn{3}{|c|}{ Bottom 6/14 } \\
\hline RAE 2001 & RAE 2008 & REF 2014 & RAE 2001 & RAE 2008 & REF 2014 \\
\hline Birkbeck & Imperial College & Birmingham & St Martin's & Goldsmiths & Chichester \\
\hline Cambridge & Essex & York & St Mary's & Sheffield Hallam & Newman \\
\hline Durham & Kent & Sheffield & Westminster & Leeds TAS & Chester \\
\hline East Anglia & Liverpool & Southampton & Worcester & Canterbury CC & Westminster \\
\hline King's College & Oxford & Hertfordshire & York & Chichester & Ctrl. Lancashire \\
\hline SOAS & Warwick & King's College & Glamorgan & Cumbria & Liverpool Hope \\
\hline Oxford Brookes & Cambridge & Warwick & Bath Spa & Westminster & Leeds Trinity \\
\hline LSE & UCL & Oxford & Bolton & Gloucestershire & Greenwich \\
\hline Birmingham & Birkbeck & Exeter & Chester & Liverpool JMs & St Mary's \\
\hline Essex & Southampton & Cambridge & Edge Hill & Edge Hill & Bath Spa \\
\hline Exeter & Hertfordshire & Manchester & Liverpool Hope & Northumbria & Sunderland \\
\hline Hertfordshire & LSE & Leeds & Middlesex & Newman College & Anglia Ruskin \\
\hline Huddersfield & Sheffield & St Andrews & Staffordshire & Wales, Newport & Gloucestershire \\
\hline Hull & Aberdeen & UCL & Thames Valley & Worcester & B. Grosseteste \\
\hline
\end{tabular}

Auf den ersten Blick deutet sich eine relativ hohe Dynamik der Ranggruppenzusammensetzung zwischen den drei Assessments an. Es ist keineswegs so, dass in der Geschichtswissenschaft immer die gleichen Departments an der Spitze stehen. Bei genauerem Hinsehen zeigt sich jedoch eine gewisse Stabilität der Ranggruppen. Sie lässt sich beispielhaft in dreierlei Hinsicht verdeutlichen: Erstens landen immerhin 13 Departments in mindestens zwei von drei Assessments in den Top 14. Zweitens landen zehn Departments in zwei von drei Assessments in den Bottom 14. Drittens steigt in allen drei Assessments nur ein einziges Department von den Bottom 14 in die Top 14 auf, während kein einziges Department von den Top 14 in die Bottom 14 absteigt. Die oberen Ranggruppen scheinen also ein verlässlicher Garant für den Statuserhalt von Departments zu sein. Angesichts der oben zitierten Allokationslogik des RAE/REF, nach der Forschungsgelder gezielt auf "exzellente“ Departments konzentriert werden, ist das durchaus erwartungsgemäß.

Nach einem Blick auf die Ranggruppen lohnt sich ein Blick auf die Publikationsprofile dieser Ranggruppen. Hierbei konzentriere ich mich der Übersichtlichkeit halber auf die letzten beiden Assessments 2008 und 2014. Die von den Ranggruppen für die beiden Assessments eingereichten 
Publikationen habe ich addiert. Abbildung 2 zeigt die von den jeweiligen Ranggruppen insgesamt eingereichten Fachzeitschriftenaufsätze, Monografien und Sammelbandbeiträge.

Abbildung 2: Publikationsprofile der Ranggruppen, absolut (basierend auf für RAE 2008 und REF 2014 eingereichten Publikationen $)^{5}$

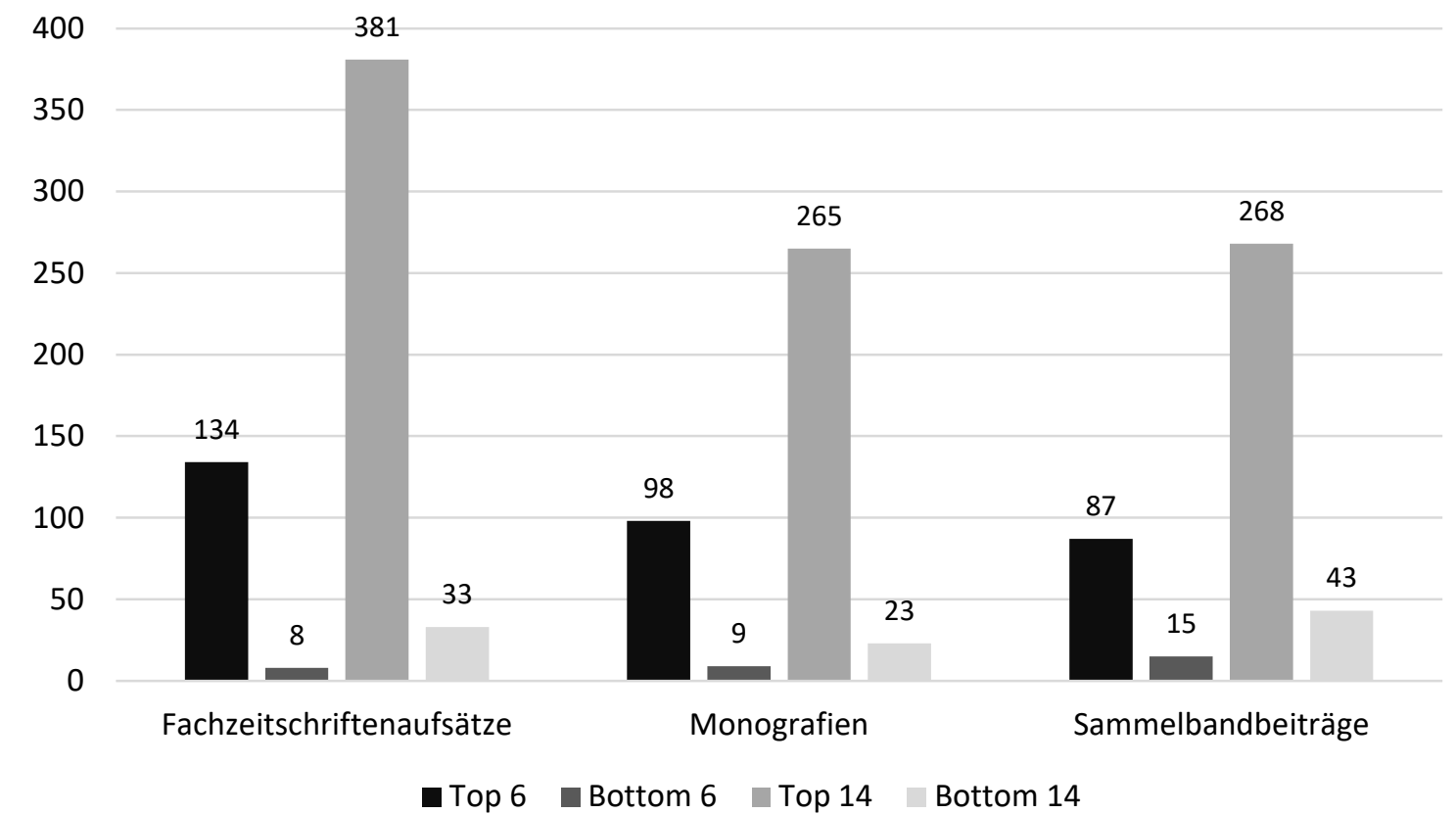

Für die Publikationsprofile wurden die von den Departments in den jeweiligen Ranggruppen des RAE 2008 und des REF 2014 eingereichten Publikationen addiert.

(eigene Berechnung nach RAE 2008b; REF 2014a)

Was in der Abbildung sofort auffällt sind die starken Unterschiede im absoluten Publikationsoutput zwischen den oberen und unteren Ranggruppen. Dazu komme ich noch. Zunächst ist ein anderer Aspekt hervorzuheben: Abbildung 2 zeigt, dass die oberen Ranggruppen (Top 6/14) vor allem Fachzeitschriftenaufsätze und am wenigsten Sammelbandbeiträge zur Bewertung eingereicht haben. Die Departments in den Top 6 der Assessments von 2008 und 2014 konnten beispielsweise insgesamt 134 Fachzeitschriftenaufsätze, 98 Monografien und 87 Sammelbandbeiträge einreichen. Für die unteren Ranggruppen (Bottom 6/14) ist das Gegenteil richtig: Sie haben vor allem Sammelbandbeiträge eingereicht und am wenigsten Fachzeitschriftenaufsätze. Die Departments in

\footnotetext{
${ }^{5}$ Die eingereichten Publikationen wurden für die Berechnung noch einmal qualifiziert, indem nur insgesamt 126 high impact-Fachzeitschriften und 19 prestigeträchtige Buchverlage zugrunde gelegt wurden. Diese Auswahl ist informiert durch meine eigenen Arbeiten zur Geschichtswissenschaft (z.B. Gengnagel \& Hamann 2014; Hamann 2014; Hamann 2016), wurde während der Erhebung überarbeitet und erweitert und ist weitgehend kongruent mit anderen Ergebnissen zum Thema (vgl. Zuccala et al. 2014).
} 
den Bottom 6 haben für die Assessments 2008 und 2014 beispielsweise insgesamt 8 Artikel, 9 Monografien und 15 Sammelbandbeiträge eingereicht.

Um die starken Unterschiede im absoluten Publikationsoutput der oberen und unteren Ranggruppen zu relativieren, können in einem weiteren Schritt die pro Kopf-Publikationen herangezogen werden (Abbildung 3). Pro Kopf meint hier nicht das gesamte Personal an den Departments, sondern ist auf das zum Assessment angemeldete Forschungspersonal bezogen.

Abbildung 3: Publikationsprofile der Ranggruppen, pro Kopf (basierend auf für RAE 2008 und REF 2014 eingereichten Publikationen)

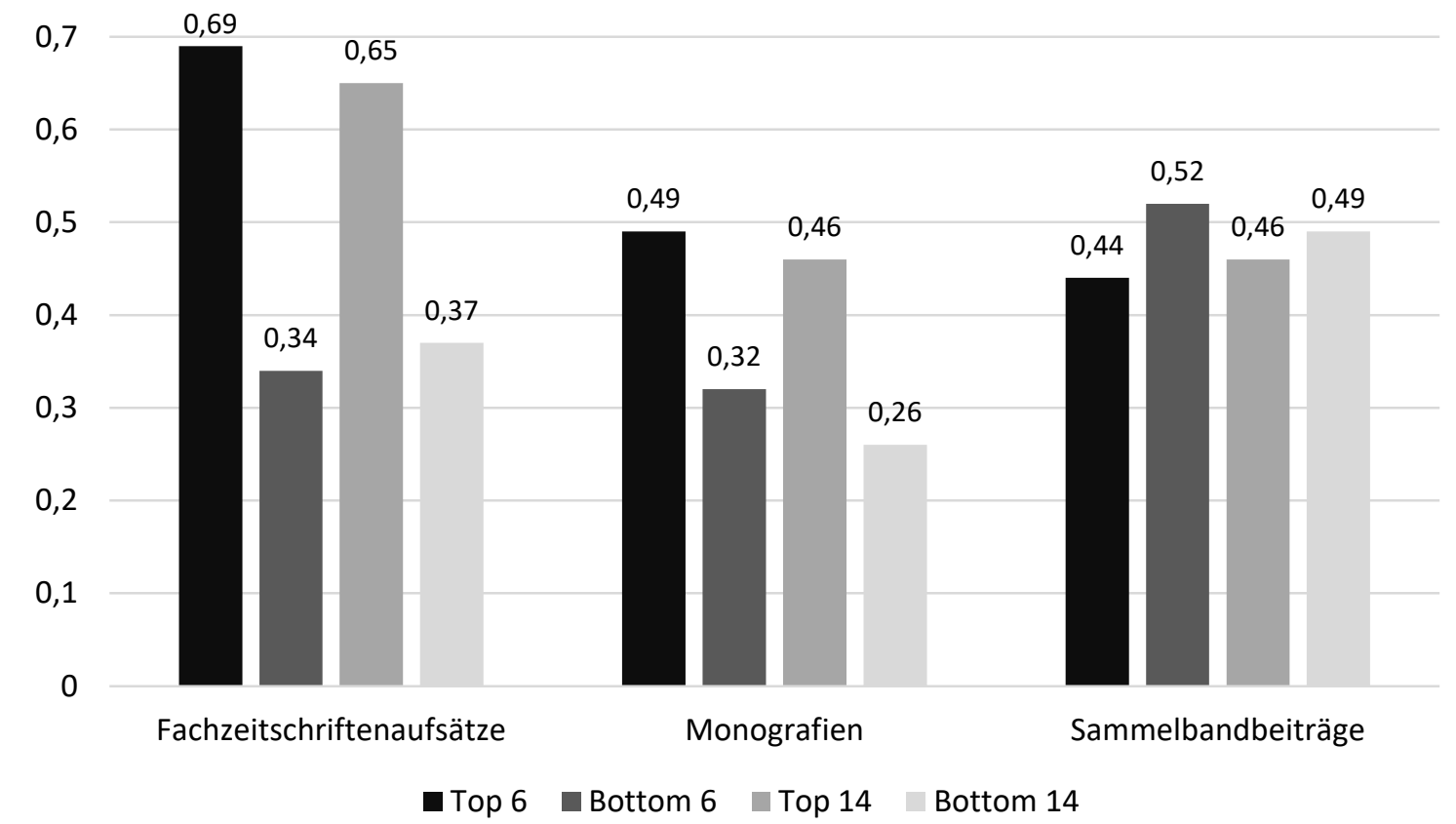

Für die Publikationsprofile wurden die von den Departments in den jeweiligen Ranggruppen des RAE 2008 und des REF 2014 eingereichten Publikationen pro Kopf addiert.

Abbildung 3 macht zunächst deutlich, dass die starken Unterschiede, die Abbildung 2 für das Publikationsoutput der oberen und unteren Ranggruppen nahelegt, nicht in erster Linie auf unterschiedliche Produktivitätsniveaus der jeweiligen Ranggruppen zurückzuführen sind, sondern auf die absoluten Unterschiede in der Anzahl der Forscher*innen. Damit wird klar, wie stark Größeneffekte die Sichtbarkeit von Departments und ihre Präsenz in Publikationsorganen bestimmen (Münch 2008). Trotz der Größeneffekte bleiben die zuvor festgestellten Muster bestehen: Abbildung 3 zeigt, dass die Departments in den oberen Ranggruppen pro Kopf mehr Fachzeitschriftenaufsätze und Monografien zur Bewertung einreichen konnten. So haben die Departments in den Top 6 für beide Assessments pro 
Kopf insgesamt 0,69 Fachzeitschriftenaufsätze und 0,49 Monographien eingereicht. Für die unteren Ranggruppen ist erneut das Gegenteil richtig: Sie haben auch pro Kopf mehr Sammelbandbeiträge als die oberen Ranggruppen eingereicht. Keine andere Ranggruppe hat 2008 und 2014 mehr Sammelbandbeiträge pro Kopf zur Bewertung eingereicht als die Bottom 6.

Als Zwischenfazit kann also festgehalten werden, dass hohe Ranggruppen mit einer Publikationsstrategie einhergehen, die stark auf Fachzeitschriftenaufsätze und wenig auf Sammelbandbeiträge konzentriert ist. Niedrige Ranggruppen gehen mit einer geradezu spiegelverkehrten Publikationsstrategie einher, die einen schwachen Fokus auf Fachzeitschriftenaufsätze und einen starken Fokus auf Sammelbandbeiträge hat. Zur Interpretation der Befunde gehört auch hervorzuheben, dass es sich hier um Korrelationen und nicht um Kausalitäten handelt. Zukünftige Forschung wird sich damit auseinandersetzen müssen, inwiefern kausale Zusammenhänge bestehen - und in welche Richtung diese Kausalitäten zeigen, ob also spezifische Publikationsstrategien zu entsprechenden Ranggruppen führen oder ob Ranggruppen über verschiedene Runden des Assessments hinweg zu entsprechenden Publikationsstrategien führen.

\section{Diskussion: Generische Leistungsbewertung als Motor für disziplinäre Entdifferenzierung?}

Die präsentierten Befunde legen nahe, dass es einen klaren Zusammenhang zwischen der Leistungsbewertung des RAE/REF und spezifischen Publikationsformen gibt. Auch wenn offen bleibt, ob es sich hier um einen kausalen Zusammenhang handelt, lässt sich auf Grundlage der Ergebnisse argumentieren, dass das britische Assessment Aufsätze in high impact-Fachzeitschriften durch Sichtbarkeit, Prestige und Forschungsgelder auszeichnet. Damit wird eine Publikationsform prämiert, die der Geschichtswissenschaft zwar keineswegs völlig fremd ist, dort aber wohl nicht den hohen Stellenwert hat, der ihr im Assessment zukommt. Monografien, die in der Geschichtswissenschaft einen traditionell hohen Stellenwert haben, werden vom Assessment ebenfalls belohnt, allerdings in einem geringeren Ausmaß als Fachzeitschriftenaufsätze. Die Publikation von Sammelbandbeiträgen, traditionell eigentlich ein fester Bestandteil der geschichtswissenschaftlichen Publikationskultur, wird vom Assessment offensichtlich nicht prämiert. Es liegt nahe, dass die vom RAE/REF belohnte spezifische Publikationskultur mit ihrem Fokus auf Aufsätzen in high impact-Fachzeitschriften naturwissenschaftlicher Prägung ist.

Es spricht also viel dafür, dass eine Leistungsbewertung, die erstens generisch auf alle Disziplinen angewendet wird und zweitens durch Sichtbarkeit, Prestige und Forschungsgelder eine enorme Wirksamkeit entfaltet, mit der Einebnung und Entdifferenzierung fachspezifischer Publikationskulturen einhergeht. 
Abschließend sollte dieser Befund noch in zwei Punkten qualifiziert werden: Erstens findet die hier angedeutete Entdifferenzierung fachspezifischer Publikationskulturen keineswegs unter dem Vorzeichen disziplinärer Indifferenz statt. Für das RAE/REF spielen disziplinäre Unterteilungen durchaus eine große Rolle. Die UoAs sind ein für das Bewertungsverfahren zentrales Sortierungsraster, das dezidiert aus einer disziplinären Logik heraus gedacht ist. Dies führt so weit, dass sich Forschung, die quer zu diesen disziplinären Unterteilungen liegt, nur schwer behaupten kann (Stern 2016: 15). Der Motor für die disziplinäre Entdifferenzierung scheint also weniger die generelle Auflösung von Fachkulturen zu sein. Ausschlaggebend ist vielmehr die Tatsache, dass spezifische, dominante Fachkulturen - zu vermuten ist: solche aus den Naturwissenschaften - andere Fachkulturen ergänzen und teilweise überschreiben. Die Entdifferenzierung der geschichtswissenschaftlichen Publikationskultur erfolgt also in Richtung naturwissenschaftlicher Publikationskulturen.

Die zweite Qualifizierung des Ergebnisses ist, dass die Entdifferenzierung einer geschichtswissenschaftlichen Publikationskultur mit einer Differenzierung innerhalb der Geschichtswissenschaft einhergeht. Die Disziplin wird durch die Leistungsbewertung des RAE/REF nachhaltig stratifiziert. Mit dem Assessment bildet sich eine „exzellente“, sichtbare, bestens mit Forschungspersonal und Forschungsgeldern ausgestattete Spitze aus (Hamann 2018). Dieser Spitze stehen große Teile des Feldes gegenüber, die geringe Aussichten auf den Exzellenzstatus haben und womöglich auch gar nicht danach streben. Sie verfolgen entweder andere, traditionell geschichtswissenschaftliche Publikationsstrategien oder nehmen gar nicht am Assessment teil, um angesichts geringer Erfolgschancen die Kosten des mit einer Teilnahme einhergehenden Berichtswesens zu vermeiden. Diese Departments bleiben in den Rankings und in der öffentlichen Berichterstattung unsichtbar. Es ist diese Differenzierung des Feldes in "exzellente" Spitzendepartments, abgeschlagene untere Ranggruppen und unsichtbare Departments, die mit der beschriebenen Entdifferenzierung einhergeht.

Offen bleibt, was die Entdifferenzierung der geschichtswissenschaftlichen Publikationskultur im Rahmen der Leistungsbewertung für die Wissensproduktion in der Disziplin bedeutet. Sehen wir hier nur eine oberflächliche Anpassung, von der die Forschungsaktivitäten und -inhalte nicht berührt sind? Zu dieser These würde die Eingangs zitiere Literatur zur Reaktivität im Sinne einer oberflächlichen, strategischen Anpassung an Bewertungskriterien tendieren. Denkbar ist aber auch, dass sich mit der Entdifferenzierung der geschichtswissenschaftlichen Publikationskultur auch die Forschungsaktivitäten und -inhalte auf kleinteiligere, schneller getaktete Einheiten umstellen. Zur Bearbeitung dieser Frage soll der vorliegende Beitrag ermutigen. 


\section{Literatur}

Biagioli M, et al. 2019. Academic misconduct, misrepresentation, and gaming: A reassessment. Research Policy 48(2): 401-413.

Campbell DT. 1979. Assessing the impact of planned social change. Evaluation and Program Planning 2(1): 67-90.

Campbell K, et al. 1999. Journal Publishing, Journal Reputation, and the United Kingdom's Research Assessment Exercise. Journal of Law and Society 26(4): 470-501.

Derrick GE \& Samuel GN. 2016. The Evaluation Scale: Exploring Decisions About Societal Impact in Peer Review Panels. Minerva 54(1): 75-97.

Espeland WN \& Sauder M. 2007. Rankings and reactivity. How public measures recreate social worlds. American Journal of Sociology 113(1): 1-40.

Gengnagel V \& Hamann J. 2014. The Making and Persisting of Modern German Humanities. Balancing Acts between Autonomy and Social Relevance. In: Bod R et al. (Hg.) The Making of the Humanities III. The Modern Humanities. Amsterdam: Amsterdam University Press, 641-654.

Hamann J. 2014. Die Bildung der Geisteswissenschaften. Zur Genese einer sozialen Konstruktion zwischen Diskurs und Feld. Konstanz: UVK.

Hamann J. 2016. 'Let us salute one of our kind'. How academic obituaries consecrate research biographies. Poetics 56(3): 1-14.

Hamann J. 2018. The production of research elites. Research performance assessment in the United Kingdom. In: Bloch R et al. (Hg.) Universities and the Production of Elites. Discourses, Policies, and Strategies of Excellence and Stratification in Higher Education. New York: Palgrave Macmillan, 175-199.

Hamann J, et al. 2019. Ist gesellschaftliche Relevanz von Forschung bewertbar, und wenn ja, wie? Wien: Österreichische Akademie der Wissenschaften.

Hammarfelt B \& de Rijcke S. 2015. Accountability in context: effects of research evaluation systems on publication practices, disciplinary norms, and individual working routines in the faculty of Arts at Uppsala University. Research Evaluation 24(1): 63-77.

Jehne M. 2009. Publikationsverhalten in den Geschichtswissenschaften. In: Alexander von HumboldtStiftung (Hg.) Publikationsverhalten in unterschiedlichen wissenschaftlichen Disziplinen. Bonn: $\mathrm{AvH}$, 59-61.

Krücken G, et al. 2021. Multipler Wettbewerb im Hochschulsystem - Interdisziplinäre Perspektiven und wissenschaftspolitische Implikationen. Das Hochschulwesen, im Erscheinen 2021(3).

Lee FS, et al. 2013. The UK Research Assessment Exercise and the narrowing of UK economics. Cambridge Journal of Economics 37(4): 693-717.

Marginson S. 2015. UK Research Excellence: Getting Better All the Time? International Higher Education 81(2015): 12-13.

Münch R. 2008. Stratifikation durch Evaluation. Mechanismen der Konstruktion und Reproduktion von Statushierarchien in der Forschung. Zeitschrift für Soziologie 37(1): 60-80.

Münch R \& Schäfer LO. 2014. Rankings, Diversity and the Power of Renewal in Science. A Comparison between Germany, the UK and the US. European Journal of Education 49(1): 60-76.

RAE. 1992. Universities Funding Council. Research Assessment Exercise 1992: The Outcome. Circular 26/92 Table 62, History. Available at: http://www.rae.ac.uk/1992/c26 92t62.html.

RAE. 1996. 1996 Research Assessment Exercise, Unit of Assessment: 59 History. Available at: http://www.rae.ac.uk/1996/1 96/t59.html.

RAE. 2001a. 2001 Research Assessment Exercise. Unit of Assessment: 59 History. Available at: http://www.rae.ac.uk/2001/results/byuoa/uoa59.htm.

RAE. 2001b. A Guide to the 2001 Research Assessement Exercise. Available at: http://www.rae.ac.uk/2001/pubs/other/raeguide.pdf.

RAE. 2008a. RAE 2008 quality profiles UOA 62 History. Available at: http://www.rae.ac.uk/results/qualityProfile.aspx?id=62\&type=uoa. 
RAE. 2008b. RAE 2008 submissions, UOA 62 History. Available at: http://www.rae.ac.uk/submissions/submissions.aspx?id=62\&type=uoa.

REF. 2012. Panel criteria and working methods, Part 2D: Main Panel D criteria. Available at: https://www.ref.ac.uk/2014/media/ref/content/pub/panelcriteriaandworkingmethods/01 1 2 2D.pdf.

REF. 2014a. REF 2014 Results \& submissions, UOA 30 - History. Available at: http://results.ref.ac.uk/Results/ByUoa/30.

REF. 2014b. Results and submissions. Available at: http://results.ref.ac.uk/Results.

REF. 2014c. View results and submissions by UOA. Available at: http://results.ref.ac.uk/Results/SelectUoa.

Sharp S \& Coleman S. 2005. Ratings in the Research Assessment Exercise 2001 - the Patterns of University Status and Panel Membership. Higher Education Quarterly 59(2): 153-171.

Sivertsen G. 2017. Unique, but still best practice? The Research Excellence Framework (REF) from an international perspective. Palgrave Communications 3(17078): 1-6.

Stern N. 2016. Research Excellence Framework (REF) review: Building on success and learning from experience. London: Department for Business, Energy \& Industrial Strategy.

Tapper T \& Salter B. 2002. The External Pressures on the Internal Governance of Universities. Higher Education Quarterly 56(3): 245-256.

Technopolis Group. 2018. Review of the Research Excellence Framework. Brighton: Technopolis Group.

The Guardian. 2014. University Research Excellence Framework 2014 - the full rankings. Available at: https://www.theguardian.com/news/datablog/ng-interactive/2014/dec/18/universityresearch-excellence-framework-2014-full-rankings.

Weingart P, et al. 1991. Die sog. Geisteswissenschaften: Außenansichten. Frankfurt/M.: Suhrkamp.

Whitley RD, et al. (Hg.). 2010. Reconfiguring Knowledge Production. Changing Authority Relationships in the Sciences and their Consequences for Intellectual Innovation. Oxford: Oxford University Press.

Yokoyama K. 2006. The effect of the research assessment exercise on organisational culture in English universities: collegiality versus managerialism. Tertiary Education and Management 12(4): 311-322.

Zuccala A, et al. 2014. Can We Rank Scholarly Book Publishers? A Bibliometric Experiment with the Field of History. Journal of the American Society for Information Science and Technology 66(7): 1333-1347. 